\title{
Getting on my nerves
}

Several cancers metastasize to bone, leading to pain that can substantially influence patients' quality of life. However, little is known about the molecular mechanisms behind this cancer-associated pain. Rohini Kuner and colleagues provide evidence implicating two receptor tyrosine kinases in this process.

The authors began with the observation that higher levels of secreted granulocyte and granulocytemacrophage colony-stimulating factors (G-CSF and GM-CSF) are found in biopsies from patients with pancreatic adenocarcinoma than biopsies from normal pancreas. When using immunohistochemistry to analyse the expression of the

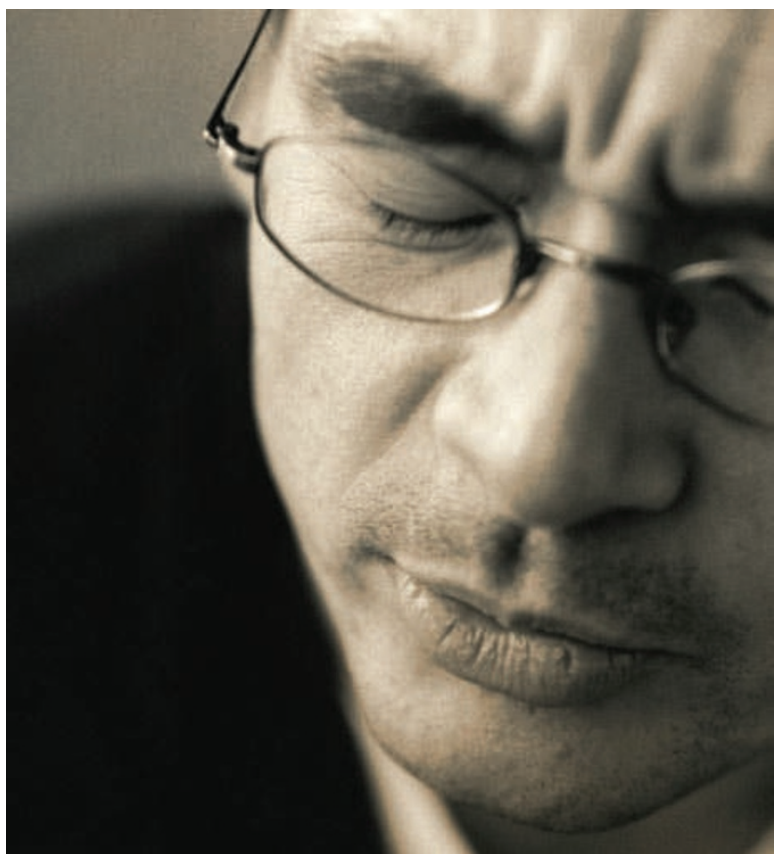

receptors for G-CSF (G-CSFR) and GM-CSF (GM-CSFR $\alpha$ ), they unexpectedly found these receptors on the nerves that innervate the pancreas - they are typically localized to haematopoietic cells. Furthermore, in a mouse sarcoma model of bone tumour-induced pain, tissue near the tumour had high levels of G-CSF and GM-CSF, and G-CSFR and GM-CSFR $\alpha$ were expressed on sensory nerves. These receptors were shown to be functional, and several lines of evidence suggested that they might affect pain perception through the sensitization of peripheral nociceptive nerves (nerves that respond to noxious stimuli that are likely to cause tissue damage by eliciting pain). Mice with sarcomas on their heel bone had hyperalgesia (increased sensitivity to pain) in response to tactile stimulation, but sham-stimulated mice did not. Injection of neutralizing antisera against either G-CSFR or GM-CSFR $\alpha$ prevented hyperalgesia, whereas control immunoglobulin $\mathrm{G}$ injections had no effect.

How might signalling through G-CSFR and GM-CSFRa induce cancer-related pain? G-CSFR and GM-CSFRa signal through the Janus kinase (Jak)-signal transducer and activator of transcription (Stat) pathway and can crosstalk with the Erk and PI3K pathways. Inhibitors of either the Jak family (AG490) or MEK (PD98059) also inhibited hyperalgesia, but the PI3K inhibitor LY294002 did not. Mice treated with G-CSFR or GM-CSFRa antisera had less local nerve branching and sprouting in the skin overlying the tumour than mice treated with immunoglobulin G; inhibition of G-CSFR or GM-CSFR $\alpha$ also reduced tumour size.

To show that the observed effects on nerve structure and pain reduction were independent of any effects of G-CSFR and GM-CSFR $\alpha$ inhibition on tumour growth, the authors directly injected lentiviruses expressing short hairpin RNAs (shRNAs) against Csf2ra (which encodes GM-CSFR $\alpha$ )into dorsal root ganglion neurons in mice prior to inducing tumours. Csf2ra shRNAs inhibited hyperalgesia, indicating the importance of this receptor in sensory neurons. Furthermore, the nerve branching that was observed in control mice was reduced in response to Csf2ra shRNAs; cultured dorsal root ganglion neurons, which were free from tumour cells, also exhibited increased branching in response to GM-CSF, indicating that these growth factors can contribute to the remodelling of nerve structure.

In addition to pointing to possible therapeutics to reduce pain in cancer patients, these data may help explain the clinical observation that patients treated with G-CSF or GM-CSF prior to haematopoietic stem cell collection, myelosuppressive chemotherapy or radiotherapy often experience bone pain as a side effect.

Sarah Seton-Rogers

\section{ORIGINAL RESEARCH PAPER}

Schweizerhof, M. et al. Hematopoietic colony-

stimulating factors mediate tumor-nerve

interactions and bone cancer pain. Nature Med. 7 Jun 2009 (doi:10.1038/nm.1976) 